\title{
Determinants in Pay-What-You-Want Pricing Decisions-A Cross-Country Study
}

\author{
Tim Dorn1, Augustin Suessmair ${ }^{2}$ \\ ${ }^{1}$ University of Cologne, Cologne, Germany \\ ${ }^{2}$ Leuphana University of Lueneburg, Lüneburg, Germany \\ Email: tim.dorn@ymail.com, suessmair@uni.leuphana.de
}

How to cite this paper: Dorn, T. and Suessmair, A. (2017) Determinants in PayWhat-You-Want Pricing Decisions-A Cross-Country Study. American Journal of Industrial and Business Management, 7, 115-142.

https://doi.org/10.4236/ajibm.2017.72010

Received: December 28, 2016

Accepted: February 25, 2017

Published: February 28, 2017

Copyright $\odot 2017$ by authors and Scientific Research Publishing Inc. This work is licensed under the Creative Commons Attribution-NonCommercial International License (CC BY-NC 4.0). http://creativecommons.org/licenses/by-nc/4.0/

\begin{abstract}
Pay-what-you-want (PWYW) pricing has attracted much attention recently. Current research focused on influencing factors and their power across social contexts and countries. This article empirically examines a comprehensive list of 10 empirically tested factors that influence individual price-setting but never have been investigated holistically. Results indicate: (1) some previously assumed influencing factors have stronger moderating effects than do others, and (2) these influences must be interpreted as influential clusters rather than individually, as not all constructs are of significantly differing influence on an individual's pricing decision. Satisfaction (Cluster 1), price consciousness, and fairness (both Cluster 2) constitute the most crucial moderators, regardless of context. Four country contexts (i.e., Australia, Germany, Poland, United States) revealed further insights about the intercultural perspective of PWYW pricing: Whereas most of the influencing factors/clusters are of comparable importance across the tested countries, loyalty and reputation (Cluster 6) significantly differed in importance depending on country.
\end{abstract}

\section{Keywords}

Pay-What-You-Want, Willingness-to-Pay, Participative Pricing, Social Influence, Pricing Decisions, Cross-Country-Study

\section{Introduction}

Price, one of the four pillars of the marketing mix, always has played a significant role in both marketing theory and practice. As pointed out by Ahmetoglu, Furnham and Fagan [1], the use of various pricing strategies has numerous advantages for a company and marketers as they can manipulate such pricing designs to influence buyers' perceptions and purchase decisions. Somervuori's [2] summary of several studies shows that pricing research in the past has mainly focused on the 
strategic aspects associated with pricing, pricing economics, strategic pricing options, or the degree of influence of pricing research on marketing. However, this research still lacks a core understanding of the behavioral foundations that ultimately determine pricing behavior. Ahmetoglu et al. [1] further states that retailers increasingly use pricing strategies that are rooted in psychology to compete on price and thus create a competitive advantage. Therefore, behavioral pricing and the underlying psychological motives of pricing decisions from a customer's perspective have received increased scientific attention in the past (also see, e.g., Campbell [3]; Voelckner and Hofmann [4]; Xia, Monroe and Cox [5]). One of the frameworks in behavioral pricing that has been heavily investigated is participative pricing mechanisms. The most extreme form of participative pricing is pay-whatyou-want (PWYW) pricing. Kim, Natter and Spann [6] defined PWYW as a pricing mechanism in which the complete process of price determination was delegated to the buyer, therefore, giving full control over the pricing to buyers and enabling them to set any price they wished to pay for a product, requiring no minimum [7]. The sole role of sellers in this relationship is to decide to offer products under PWYW conditions, as they cannot reject but must accept the buyer's set price [8]. In combination, these two distinctive features-the buyer as the active participant in setting the price and the seller engaging in this process without imposing a threshold price and being obliged to accept any price-differentiate PWYW pricing from other participative pricing strategies. Potential benefits of this innovative pricing strategy range from attracting new customers [9] [10], which may affect image perceptions and attitudes toward the seller in a positive way [11], moderation of perceptions of price (un)fairness [12], neutralization of switching costs [13], or enablement of price discrimination toward various consumer segments [14] [15].

As pointed out by Kim et al. [6] an economically rational customer might be expected to exploit the mechanism by paying a price of zero and thus maximizing the individual purchase utility. ${ }^{1}$ However, empirical evidence e.g. Gneezy, Nelson and Brown [16], Johnson and Cui [17] and Kim et al. [6], has shown that almost no customer pays a price of zero in any kind of PWYW-setting. Greiff, Egbert and Xhangolli [18] referred to several theories, such as preferences for fairness, reciprocity or the availability of reference prices to explain this-from a mere economic perspective-irrational kind of behaviour, and Kim et al. [6] also named various behavioral aspects that influence PWYW pricing decisions.

Because of the relative novelty of PWYW, numerous examinations of the pricing mechanism in terms of associated fairness considerations [19], its competitiveness [11] [20] [21], or related social norms [7] [16] have recently been published. However, no holistic composition of the underlying influences of a PWYW decision has been developed yet. Although there exists sufficient empirical evidence that several psychological constructs influence PWYW pricing decisions [9] [22] and factors such as fairness and reciprocity concerns play a crucial role in

${ }^{1}$ The most extreme form of a negative price, which gives the consumer the power even to punish the seller, has not been researched yet. 
PWYW interactions [6] [23] [24], attempts to examine the relative importance of the influential factors have been relatively rare, e.g., Dorn and Suessmair [22], Kunter [23] and are still rudimentary. Hence, the academic literature is even sparser on the intercultural dimension of PWYW pricing and research is needed to grasp and assess intercultural differences as well as commonalities of PWYW decisions.

This article examines the relative importance of the determinants of PWYW pricing decisions. To do so, all empirically proven influential constructs of a PWYW decision are examined in an empirical study. Additionally, the intercultural components of PWYW decisions are addressed, as the study was simultaneously conducted in Germany, Australia, Poland, and the United States. Ultimately, this study will show to what extent a consumer's PWYW pricing decision is influenced by any of the previously researched influential determinants and refine this finding from both a situational and an intercultural point of view.

\section{Theoretical Foundation}

When speaking of influences on PWYW pricing, it is important to differentiate between three kinds of influences:

1) Influences on consumer behavior that are triggered by the sheer fact that the pricing mechanism is offered; these influences arise because of the pricing mechanism itself and exist inherently;

2) Classical instruments of organizational design that sellers can use during the set up and the offer period of the PWYW quotation to influence a customer's willingness to pay $\left(\mathrm{WTP}^{2}\right)$; and

3) Influential determinants that are subjective and individual for each customer.

Each customer builds up such determinants individually and sellers can affect these determinants only to a certain degree but never holistically.

As the first and second type of influences have been examined in the research fairly extensively [6] [10], this article focuses upon the third, 10 empirically proven influential determinants: Satisfaction [6] [15], Income [6] [22] [25], Price Consciousness (PC) [6] [7] [25], Reference Price (RP) [24] [26] [27], High Level of Reputation (HLoR) [11], Loyalty [7] [9] [25], Altruism [25] [26] [28], Fairness ${ }^{3}$ [3] [5] [12] [28], Social Acceptance (SA) [9] [11] [29], Social Norm Compliance (SNC) [11] [23] [30] [31].

\footnotetext{
${ }^{2}$ As elaborated by Miller et al. (2011) [38] the concept of WTP refers to the maximum amount of money at which or below which a consumer would be willing to buy the presented product.

${ }^{3}$ In this context, it is important to differentiate between fair behavior by the company toward the customer and fair behavior by the customer toward the company. Relative to fair corporate behavior, perceived price fairness plays a major role in the academic literature and displays a relatively well-investigated construct. Indeed, it is important to understand how the consumer's perception of price fairness is formed, as one's perception of being treated fairly and paying a fair price constitutes one of the basics of why PWYW pricing works-see also Haws and Bearden [48] and Kim et al. [6]. However, as this article deals with the determinants, which influence a consumer's decision under PWYW circumstances, fairness considerations from a corporate perspective toward the customer are not the main object of examination. Thus, this examination of fairness primarily focuses on fairness considerations from the customer's point of view toward the company that offers PWYW and its influences on the prices paid in PWYW situations, beginning with an examination of why customers decide to respond fairly to PWYW offers, which is mainly a response to perceived price fairness.
} 


\section{Research Question}

Gerpott [32] provided a comprehensive overview of empirical studies in the field of participative pricing. Based on that overview, the present study is relevant for several reasons. Cheema and Dholakia [33] pointed out the need for further research on the behavioral factors of pricing decisions in participative pricing environments. Also, Voelckner [34] mentioned the need for an examination of the cognitive processes of consumers while forming their WTP. Although much such research has been conducted since then, the behavioral basis of PWYW pricing is far from holistically assessed. Accordingly, Kunter [23] questioned the originality and the completeness of the six influential determinants elaborated by Kim et al. [6], as they were not derived from a conceptual framework. He further added that other constructs also play a decisive role in a PWYW context. As results of studies on these influences have been ambiguous so far, he called for studies with more generalizable results. As no study has examined all 10 influential determinants holistically, this study will be the first to do so. Moreover, Kunter [23] also pointed out the importance of understanding the influential determinants and their relevance to customers' decisions as a way to learn more about the manageability of PWYW pricing and the motivations behind these decisions. Dorn and Suessmair [22] emphasized the importance of further study of the relative importance of the influential determinants.

Additionally, Armstrong Soule and Madrigal [25] referred to the importance of examining PWYW pricing with varying levels of social interaction, especially focusing on anonymous PWYW payments, as these have not been investigated thoroughly. Kunter [23] shared this opinion and indicated the importance of further research on social pressure in PWYW settings.

Thus, the topic of situational influences and accordingly the level of social interaction or social distance between seller and buyer offer a promising perspective in the examination of PWYW pricing.Gautier and van der Klaauw [35] addressed the potential threat of anonymity by stating that missing physical presence might lead to a decreased WTP in participative pricing environments. Also, Kim et al. [6] referred to the importance of personal interaction for the feasibility of the PWYW model and Armstrong Soule and Madrigal [35] stated that face-to-face transactions in PWYW are less risky than impersonal PWYW adaptations. Marett et al. [7] assumed that an insufficient level of social presence in PWYW situations might lead to a decreased WTP, and Kim et al. [9] further showed that the type of interaction between seller and buyer can influence PWYW transactions. These empirical findings are the basis for the first hypothesis:

H1: The customer's WTP increases with the level of social interaction in the respective situation.

Thus, the hypothesis wants to examine, whether customers intend to pay more when the level of social interaction during the pricing process increases or are indifferent to the level of social interaction while indicating their WTP.

Research on the influential determinants of PWYW decisions has produced divergent results so far. Although Kim et al. [6] found fairness, satisfaction, PC 
and income to be the main drivers of PWYW decisions, Dorn and Suessmair [22] showed a significant influence of SA and León et al. [26] pointed out the importance of internal reference prices. Whereas most of the studies have shown some overlap of influences, some determinants have been mentioned more frequently than others and their influence on pricing decisions under PWYW conditions is more obvious. Thus, it can be hypothesized that these determinants have a more apparent or even relatively greater influence on PWYW decisions. Moreover, Kim et al. [6] ${ }^{4}$ set up an estimation model to explain prices set by buyers in PWYW transactions in which various coefficient estimations were found for the influential determinants, thus signaling a different relative importance of the constructs. Therefore, the second hypothesis assumes that:

H2: Not all influential determinants are of the same importance to pricing decisions in PWYW settings; some determinants influence PWYW decisions more significantly than others.

By means of this, the second hypothesis tries to grasp the different importance subjects are going to attribute to the various influential constructs.

As the present study was conducted in an international environment, the interculturality of a PWYW decision is discussed. Most of the studies that have focused on the influential determinants of a PWYW decision have been made by a variety authors and been conducted in various countries. Nonetheless, these studies have produced comparable results about the existence and the content of influential determinants. Thus, it can be assumed that the influential determinants of a PWYW decision are interculturally stable and comparable. However, Mills [36] revealed an explicit heterogeneity of the buyer's WTP in PWYW settings in an intercultural study, showing that customers from different cultures paid different prices in PWYW scenarios. Also, Weisstein, Siew and Monroe [37] found evidence for differing consumer behavior in a participative pricing context from an intercultural viewpoint. Thus, if individuals, regardless of their cultural background, are influenced by identical determinants when making a pricing decision under PWYW circumstances, but cross-cultural studies show different results for WTP and consumer behavior, we come to the third hypothesis of this study:

H3: The relative importance of influential determinants differs between participants from different countries.

This hypothesis suggests that subjects from different countries are going to rank the influential constructs when making a PWYW decision differently. Thus, some influential determinants are going to become relatively more important for subjects from one country than to subjects from another country.

\section{Methodology}

Because the study was conducted in an international environment among several countries, the use of a questionnaire was the most feasible approach for data ac-

${ }^{4}$ This equation model includes all of the influential determinants examined in their respective study (fairness, altruism, loyalty, satisfaction, PC, income and reference price). 
quisition. Because the study represents a laboratory experiment and was not carried out in field by using actual products and money, a suitable approach of how to measure the amount of money exchanged in such a hypothetical PWYW situation had to be applied. As pointed out by Voelckner [34] points out, direct price inquiries have no significant differences in their reliability from indirect price inquiries and can be regarded as one of the most practical methods by which to measure individual WTP. Also, Miller, Hofstetter, Krohmer and Zhang [38] showed that a hypothetical approach in inquiring about a consumer's WTP can be regarded as methodologically valid and may lead to accurate forecasts of demand curves. Thus, this survey used a direct inquiry of each participant's WTP that was already tested by Dorn and Suessmair [22] in a hypothetical PWYW scenario to assess the amount of money exchanged in potential PWYW situations, as used in other studies in this area, e.g., Homburg, Koschate and Hoyer [39].

One of the most crucial decisions for this research project was the selection of an adequate reference product for which participants would be asked to indicate their WTP. As intercultural aspects play a decisive role in this study, a homogeneous reference product that represents a fully processed end product ready for consumption that is standardized and equal across the tested countries had to be employed. For this purpose, McDonald's Big Mac was chosen as a reference product. The Big Mac represents a commonly used reference product for various scientific projects, the most well known being The Economists' [40] Big Mac Index.

To enable participants from each country to indicate their WTP in their home currency, an identical version of the questionnaire was set up for each country, only differing in terms of the currencies used.

As a result of these considerations, the questionnaire hypothetically referred to McDonald's offering a special promotion for the purchase of the Big Mac to mark its $100^{\text {th }}$ anniversary, enabling the customers to pay what they want for the burger. However, not only the WTP in the context of a PWYW decision is of importance in this study, but also the situational circumstances in which participants have faced PWYW decisions was considered. Therefore, three hypothetical scenarios were set up. Based upon the suggestions of Kunter [10], the scenarios varied only with regard to their social presence and observation. The scenarios were manipulated as to whether the payment was made anonymously and online (Situation 1), publicly in direct contact with the sales staff (Situation 2) or publicly in direct contact with the sales staff and under observation by a third party (Situation 3). The questionnaire presented participants with 1 of the 3 hypothetical scenarios. After a short description of the situation and the pricing process, participants were asked to indicate their WTP for the respective situation in their home currency. ${ }^{5}$ Each participant was randomly shown only 1 out of the 3 hypothetical scenarios in an adaptation of Campbell's [3] between-subjects design.

${ }^{5}$ For the exact wording of the questions as well as all other contents of the questionnaire, please refer to Web Appendix B, Questionnaire. 
After indicating their WTP for the given situation, the participants were asked to think about the potential influences that might have affected their WTP in the scenario. For this purpose, a list of the 10 previously discussed influential determinants of a PWYW decision was presented to the participants with a short description of each construct. With regard to the cultural perspective of this study, it seemed appropriate to define the constructs in advance before participants evaluated them, as this could ensure that all participants had a similar understanding of each influential determinant. These definitions were based upon earlier multi-item operationalizations of the respective constructs and were written to comprise the operationalizations as shown in Table 1.

To assess each construct's relative importance, the questionnaire used a constant sum approach: After having read each influential determinant's operationalization, the participants were asked to divide 100 points between these potential influences to show the value or importance they placed on each option. Accordingly, the more important influences were given a greater number of points; however, the total amount of points distributed always had to equal exactly 100 points. In addition to the 10 predefined influential determinants, the participants also were able to indicate further influences on their decision with a constant sum weight in a free text field at the end of the list, which produced the total list of 10 influential determinants.

\section{Implementation and Data Preparation}

As the study took place in an intercultural context, all participants received an English version of the questionnaire. For this purpose, the online questionnaire was launched on the $22^{\text {nd }}$ of July 2015 in Australia, Chile, China, Germany, Italy, Japan, Poland, Qatar, Singapore, United Arab Emirates and United States. To distribute the questionnaire, mainly mailing lists from academic institutions were used, but survey links also were shared among various academic Facebook groups and research forums in the respective countries. All surveys simultaneously were

Table 1. Sources of construct operationalization.

\begin{tabular}{cc}
\hline Construct & Literature used for operationalization \\
\hline Altruism & [Kim et al. (2009)] \\
Loyalty & [Bettencourt (1997); Kim et al. (2009)] \\
Fairness & [Campbell (1999) [3]; Kim et al. (2009)] \\
Satisfaction & [Baker, Dhruv and Parasuraman (1994); Kim et al. (2009)] \\
Price Consciousness & [Donthu and Gilliland (1996); Kim et al. (2009)] \\
[Bearden, Kaicker, Smith de Borrero and & Urbany (1992); Kim et al. (2009)] \\
Reference Price & [Coleman (1983); Kim et al. (2009)] \\
Income & {$[$ Dorn and Suessmair (2016)] } \\
Social Acceptance & [Hilbert and Suessmair (2015)] \\
Social Norm Compliance & [Kim et al. (2014)] \\
High Level of Reputation &
\end{tabular}


closed on August $25^{\text {th }}$ to ensure an identical processing period for all tested countries. Sufficiently sized samples (in terms of numbers of received questionnaires) from Australia $(n=132)$, Germany $(n=121)$, Poland $(n=103)$ and the United States $(n=123)$ were obtained. ${ }^{6}$ After preparing and adjusting the data for further evaluation $^{7}$, the final sample consisted of four independent samples, obtained in Australia ( $\mathrm{n}=131)$, Germany $(\mathrm{n}=121)$, Poland $(\mathrm{n}=101)$ and the United States ( $\mathrm{n}$ $=118)$. The average age across all samples was 25.6 years $(\mathrm{SD}=7.9)$ and overall more women than man took part in the study (women: $n=272$; men: $n=199$ ).

\section{Results-Prices Paid}

A descriptive overview of the participants' WTP by country and by situation (Table 2) provides interesting first insights on the structure of the prices paid in this study. As indicated by the average prices overall ( $\mathrm{S} 1=1.69 \$ ; \mathrm{S} 2=2.31$; $\mathrm{S} 3=$ $2.61 \$)$ and per country, free-riders only constituted a minority of all participants. Nonetheless, the high standard deviations relative to the prices paid in each scenario (S1: $\mathrm{SD}=1.64 ; \mathrm{S} 2: \mathrm{SD}=1.41 ; \mathrm{S} 3: \mathrm{SD}=1.44)$ refer to the wide spread of prices indicated in each situation.

Additionally, it can be seen that the participants' WTP increased not only in total but also for each country-situation combination respectively from S1 to S2 and from S2 to S3. These initial descriptive findings delivered strong support for

Table 2. Descriptive statistics prices.

\begin{tabular}{|c|c|c|c|c|}
\hline & Country Code & S1 Price in US \$ & S2 Price in US \$ & S3 Price in US \$ \\
\hline \multirow{3}{*}{ AUS } & $\mathrm{N}$ & 38 & 41 & 52 \\
\hline & Mean & 1.3583 & 2.4710 & 2.9329 \\
\hline & Std. Deviation & 1.26227 & 1.33759 & 1.48217 \\
\hline \multirow{3}{*}{ GER } & $\mathrm{N}$ & 47 & 37 & 37 \\
\hline & Mean & 2.2399 & 2.5275 & 2.7039 \\
\hline & Std. Deviation & 1.79893 & 0.98944 & 1.34284 \\
\hline \multirow{3}{*}{$\mathrm{PL}$} & $\mathrm{N}$ & 28 & 39 & 34 \\
\hline & Mean & 1.5147 & 2.2646 & 2.4671 \\
\hline & Std. Deviation & 1.49059 & 1.79008 & 1.23693 \\
\hline \multirow{3}{*}{ USA } & $\mathrm{N}$ & 41 & 39 & 38 \\
\hline & Mean & 1.4939 & 1.9874 & 2.2105 \\
\hline & Std. Deviation & 1.75742 & 1.36886 & 1.57494 \\
\hline \multirow{3}{*}{ Total } & $\mathrm{N}$ & 154 & 156 & 161 \\
\hline & Mean & 1.6919 & 2.3119 & 2.6114 \\
\hline & Std. Deviation & 1.64133 & 1.40674 & 1.43989 \\
\hline
\end{tabular}

${ }^{6}$ The sample sizes only refer to the number of fully completed questionnaires that were processed further. The overall sample sizes for the respective countries were: Australia: $\mathrm{n}=405$; Germany: $\mathrm{n}=$ 212; Poland: $\mathrm{n}=256$; United States: $\mathrm{n}=176$.

${ }^{7}$ Data assortment included cleaning with regard to participants who did not know McDonald's or the Big Mac; extreme outliers, which could negatively distort the structure of the prices paid; the freetext responses, in which participants could indicate further influences; and most importantly, the standardization of the WTP (indicated in the respective national currency) toward the US dollar to enable further comparisons on the basis of a common currency. 
H1, which states that the customer's WTP increases with the level of social interaction in the situation. However, it can be expected that the results of H1differ on the country level. A final summary of the descriptive statistics is provided in the box-whisker plots of Figure 1.

For the examination of H1 (the customer's WTP increases with the level of social interaction in the situation), a one-way ANOVA was executed to define the participant's WTP as the dependent variables ${ }^{8}$ and the situation as the factor. This comparison of means showed that the differences of the means of all prices paid per situation are significant $[\mathrm{F}(2,468)=15.35, p<0.05]$, thus initially confirming $\mathrm{H} 1$ (as shown in Table 3 ).

In-depth results from a post-hoc test ${ }^{9}$, revealed only two subsets (not three, as hypothesized). This finding suggests that only the WTP between Situation (S1) and S2, as well as S1 and S3 differs significantly, whereas no significant differences

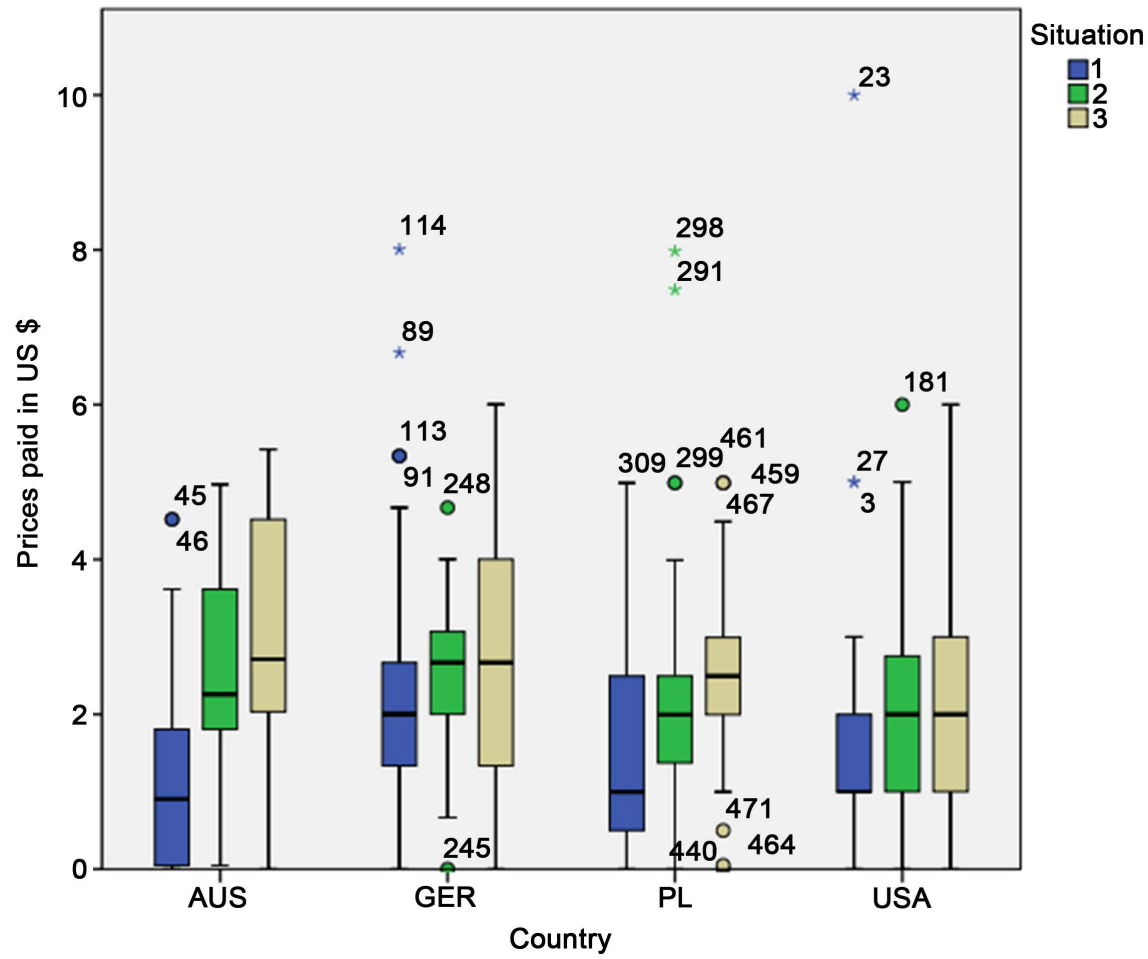

Figure 1. Box-whisker plots for the distribution of the WTP.

Table 3. ANOVAs for differences between prices paid per situation-overall and per country.

\begin{tabular}{cccccc}
\hline \multicolumn{5}{c}{ All Prices } \\
\hline & Sum of Squares & df & Mean Square & F & Sig. \\
\hline Between Groups & 68.901 & 2 & 34.451 & 15.346 & 0.000 \\
Within Groups & 1050.635 & 468 & 2.245 & & \\
Total & 1119.536 & 470 & & \\
\hline
\end{tabular}

${ }^{8}$ Overall the dependent list comprised five items: Prices paid by all subjects (All Prices) and the prices paid by subjects, separating the subjects by their country of origin (Prices USA, Prices AUS, Prices GER, Prices PL). 
between the WTP in S2 and S3 were found.

However, when examining H1from a country perspective, different results were obtained: Whereas the WTP overall increased significantly with the level of social interaction, this finding was only reproduced for Australia $[\mathrm{F}(2,128)=14.67, p<$ $0.05]$ and Poland $[F(2,98)=3.20, p<0.05]$ at the country level. The post-hoc test revealed that only the prices paid in S1 and S2 differ significantly for the Australian sample, whereas the Polish participants only showed significant differences in their WTP in S1 and S3. For American participants, the level of social influence seemingly did not play a significant role when they determined their WTP [F (2, $115)=2.15, \mathrm{~ns}]$. An additional non-parametrical test had to be executed for the German participants, as the Levene's Test indicated that the German sample does not dispose of a homogeneity of variances $[\mathrm{F}(2,118)=5.13, p<0.05]$. As this test would compare the means of the independent samples (due to the randomization of the scenarios) of the three different situations, a Kruskall Wallis H-Test was applicable. However, also the Kruskal Wallis $\mathrm{H}$-Test could not deliver significant findings for the German sample [H $(2)=4.55, p<0.05]$, thus rejecting $\mathrm{H}_{2}$ for the German and the US American sample. ${ }^{10}$

\section{Influential Determinants}

$\mathrm{H} 2$ assessed the relative importance of each construct for the PWYW decision: It was assumed that not all influential determinants would be of the same importance for a pricing decision in PWYW settings and that some determinants would influence a PWYW decision more significantly than others. A one-way ANOVA showed that the mean differences of the influential determinants were significant $[\mathrm{F}(10,5170)=87.63, p<0.05]$. As no homogeneity of variances could be assumed for this ANOVA $[\mathrm{F}(10,5170)=98.46, p<0.05]^{11}$, two further nonparametrical tests (Friedmann Test and Kendall's W Test) were performed to check the robustness of the effect. ${ }^{12}$ As both tests indicated a significant difference between the means of the influential determinants (both tests delivered identical results: $\mathrm{H}$ (10) $=1018.715, p<0.05), \mathrm{H} 2$ was confirmed (Table 4).

To elaborate further on the relative importance of the influential determinants, a post-hoc test after Duncan was used (Table 5). The results of the post-hoc test suggest the coexistence of seven groups of influence (each consisting of one or more influential determinants), each with significantly different influence. The group with the least influence comprises other (representing the entirety of all other potential influences) and loyalty. As its mean could also signal affiliation with the second influential group, the construct loyalty also is found in the next group, which consists of SA, HLoR and altruism. In line with the same reasoning (affiliation of a mean to two distinct influential groups), the third influential ${ }^{9}$ In this case, the post hoc tests after Duncan were applied.

${ }^{10} \mathrm{~A}$ detailed overview of the test results for H1can be found in the Web Appendix A-Tables 1-6d.

${ }^{11} \mathrm{~A}$ detailed overview of the test results for $\mathrm{H} 2 \mathrm{can}$ be found in the Web Appendix A-Tables 7-10.

${ }^{12}$ The Friedman test is the significance test for more than two dependent samples; it is used to test that there is no significant difference between the size of " $k$ " dependent samples and the population from which these have been drawn. Kendall's W Test is referred to the normalization of the Friedman statistic. Kendall's W is used to assess the trend of agreement among the respondents. 
Table 4. Non-parametrical checks for the robustness.

\begin{tabular}{|c|c|}
\hline \multicolumn{2}{|r|}{ Test Statistics ${ }^{\mathrm{a}}$} \\
\hline $\mathrm{N}$ & 471 \\
\hline Chi-Square & 1018.715 \\
\hline df & 10 \\
\hline Asymp. Sig. & .000 \\
\hline ªriedman Test. & \\
\hline \multicolumn{2}{|r|}{ Test Statistics ${ }^{\mathrm{a}}$} \\
\hline $\mathrm{N}$ & 471 \\
\hline Kendall'sW ${ }^{\mathrm{a}}$ & 0.216 \\
\hline Chi-Square & 1018.715 \\
\hline Df & 10 \\
\hline Asymp. Sig. & 0.000 \\
\hline
\end{tabular}

${ }^{a}$ Kendall's Coefficient of Concordance.

Table 5. Influential groups via a Duncan post hoc test.

\begin{tabular}{|c|c|c|c|c|c|c|c|c|}
\hline \multicolumn{9}{|c|}{ Duncan $^{\mathrm{a}}$} \\
\hline \multirow{2}{*}{ ConstructCode } & \multirow{2}{*}{$\mathrm{N}$} & \multicolumn{7}{|c|}{ Subsetforalpha $=0.05$} \\
\hline & & 1 & 2 & 3 & 4 & 5 & 6 & 7 \\
\hline 11-Other & 471 & 2.37 & & & & & & \\
\hline 2-Loyalty & 471 & 3.84 & 3.84 & & & & & \\
\hline 8-SocialAcceptance & 471 & & 4.36 & 4.36 & & & & \\
\hline 10-High Level of Reputation & 471 & & 5.00 & 5.00 & & & & \\
\hline 1-Altruism & 471 & & 5.14 & 5.14 & & & & \\
\hline 9-Social Norm Compliance & 471 & & & 6.02 & & & & \\
\hline 6-Reference Price & 471 & & & & 8.45 & & & \\
\hline 7-Income & 471 & & & & & 12.40 & & \\
\hline 3-Fairness & 471 & & & & & & 15.96 & \\
\hline 5-Price Consciousness & 471 & & & & & & 16.63 & \\
\hline 4-Satisfaction & 471 & & & & & & & 19.86 \\
\hline Sig. & & 0.107 & 0.199 & 0.099 & 1.000 & 1.000 & 0.466 & 1.000 \\
\hline
\end{tabular}

Means for groups in homogeneous subsets are displayed. ${ }^{a}$ Uses Harmonic Mean Sample Size $=471.000$.

cluster consists of SA, HLoR, altruism and SNC. The fourth, statistically different influence on a PWYW decision is reference price, in which income is significantly more important than other influences. The second most influential group comprises fairness and PC, and satisfaction is of significantly greater relative importance than all other influential determinants.

\section{Intercultural Aspects: Country Findings}

Ultimately, $\mathrm{H} 3$ dealt with the intercultural aspects of this study and hypothesized 
that the relative importance of influential determinants differs between participants from different cultures. For this purpose, a multivariate ANOVA was performed to define the influential determinants as the dependent variables and the country as well as the situation as fixed between-subjects factors. ${ }^{13}$

As shown in Table 6, only the influence of loyalty $[\mathrm{F}(3,459)=6.73, p<0.05]$ significantly differs between the tested countries. As this examination was limited by restrictions in terms of the homogeneity of variances, an additional Kruskal-Wallis test needed to be executed for the respective determinants (Table 7). This test showed that the effect of loyalty indeed significantly differs between the tested countries $[\mathrm{H}(3)=12.35, p<0.05]$ and also pointed out that the construct HLoR $[\mathrm{H}(3)=12.87, p<0.05]$ differs in its influence in the tested countries.

A post hoc test after Duncan further illustrated the interculturally differing influence of loyalty (Table 8), showing that the construct has a significantly stronger

Table 6. Test of between-subjects effects for country.

\begin{tabular}{|c|c|c|c|c|c|c|}
\hline \multicolumn{7}{|c|}{ Tests of Between-Subjects Effects } \\
\hline Source & Dependent Variable & $\begin{array}{c}\text { Type III Sum } \\
\text { of Squares }\end{array}$ & $\mathrm{df}$ & Mean Square & $\mathrm{F}$ & Sig. \\
\hline \multirow{11}{*}{$\begin{array}{l}\text { Country } \\
\text { Code }\end{array}$} & Altruism & 262.994 & 3 & 87.665 & 1.081 & 0.357 \\
\hline & Loyalty & 851.391 & 3 & 283.797 & 6.732 & 0.000 \\
\hline & Fairness & 1555.083 & 3 & 518.361 & 1.712 & 0.164 \\
\hline & Satisfaction & 2936.462 & 3 & 978.821 & 1.622 & 0.183 \\
\hline & Price Consciousness & 2249.104 & 3 & 749.701 & 1.964 & 0.119 \\
\hline & Reference Price & 889.162 & 3 & 296.387 & 1.941 & 0.122 \\
\hline & Income & 1860.202 & 3 & 620.067 & 2.611 & 0.051 \\
\hline & Social Acceptance & 193.772 & 3 & 64.591 & 1.275 & 0.282 \\
\hline & Social Norm Compliance & 337.525 & 3 & 112.508 & 1.353 & 0.257 \\
\hline & High Level of Reputation & 369.636 & 3 & 123.212 & 1.397 & 0.243 \\
\hline & Other & 171.051 & 3 & 57.017 & 0.467 & 0.705 \\
\hline
\end{tabular}

Table 7. Kruskal Wallis Test of the between-subjects effects (country).

\begin{tabular}{cccccccc}
\hline \multicolumn{7}{c}{ Test Statistics } \\
\hline & Loyalty & $\begin{array}{c}\text { Satis } \\
\text { faction }\end{array}$ & $\begin{array}{c}\text { Price } \\
\text { Consciousness }\end{array}$ & $\begin{array}{c}\text { Social } \\
\text { Acceptance }\end{array}$ & $\begin{array}{c}\text { Social Norm } \\
\text { Compliance }\end{array}$ & $\begin{array}{c}\text { High Level of } \\
\text { Reputation }\end{array}$ & Other \\
\hline Chi-Square & 12.345 & 7.397 & 1.602 & 2.465 & 2.960 & 12.868 & 2.546 \\
df & 3 & 3 & 3 & 3 & 3 & 3 & 3 \\
Asymp. Sig. & 0.006 & 0.060 & 0.659 & 0.482 & 0.398 & 0.005 & 0.467 \\
\hline
\end{tabular}

${ }^{\mathrm{a}}$ Kruskal Wallis Test; ${ }^{\mathrm{b}} \mathrm{Grouping}$ Variable: Country Code Numbers.

${ }^{13}$ In this case, the use of two fixed factors (situation and country) instead of calculating two one-way ANOVAs for each fixed factor separately does not change the results in terms of the findings with regard to the significance. Use of the results of the MANOVA instead of examination of each fixed factor separately (as a one-way ANOVA would do) can be regarded as the more conservative way, as it protects against inflation of the type I error rate. Moreover, use of the MANOVA for this purpose displays a more thorough depiction of the reality than a calculation of two separate ANOVAs, as interaction effects between the two fixed factors are already included even when examining the significance of each fixed factor independently (Field, [41]). A comparison of the results of the MANOVA and the according two one-way ANOVAs can be found in Web Appendix K. 
influence on participants from the United States than on those from the other tested countries. Thus, H3 could only be confirmed for loyalty and HLoR, which suggest that these two influential determinants are differ in importance on a PWYW decision interculturally, whereas the remaining eight constructs seem to represent interculturally robust influential determinants.

However, when observing the intercultural differences in the relative importance of the influential determinants from a situational perspective (i.e., examining the differences between the countries restricted to only 1 of the 3 situations), the intercultural disparities become more distinct. In this context, another one-way ANOVA, using the mean values of the influential determinants per situation as dependent variables and the country as a fixed factor, showed statistically significant differences between the countries for loyalty $[\mathrm{F}(3,150)=2.79, p<0.05]$, fairness $[\mathrm{F}(3,150)=4.56, p<0.05], \mathrm{PC}[\mathrm{F}(3,150)=3.90, p<0.05]$, income $[\mathrm{F}(3$, $150)=3.91, p<0.05]$ and $\operatorname{HLoR}[\mathrm{F}(3,150)=3.53, p<0.05]$ for S1 (Table 9). As only fairness disposed of homogeneous variances $[\mathrm{F}(3,150)=2.33$, ns], all other determinants had to be checked for robustness via a Kruskal Wallis Test (Table $10)$.

Nonetheless, this test revealed the interculturally differing influence of loyalty $[\mathrm{H}(3)=8.86, p<0.05], \mathrm{PC}[\mathrm{H}(3)=9.15, p<0.05]$ and HLoR $[\mathrm{H}(3)=12.17, p<$ $0.05]$ for $\mathrm{S} 1$ and falsified only the assumption that income $[\mathrm{H}(3)=7.68, \mathrm{~ns}]$ is of interculturally differing influence. The subsequent post-hoc test after Duncan showed that loyalty is significantly more important for Germans than for Australians for S1 (Table 11), that Polish and Germans attribute a higher relative importance to fairness than Australians and North Americans for S1 (Table 12), that Australians are significantly more price-conscious than Germans and North Americans for S1 (Table 13) and that North Americans are significantly more influenced by the HLoR than Germans, Australians and Polish people in anonymous PWYW settings (Table 14).

Whereas the findings regarding the intercultural differences of the relative importance of influential determinants for S1 have been quite distinct, similar

Table 8. Post Hoc Tests after Duncan for an assessment of the differences between the influential determinant loyalty per country.

\begin{tabular}{cccc}
\hline \multicolumn{5}{c}{ Loyalty } & \\
\hline & Duncan & & \\
\hline Country Code & $\mathrm{N}$ & \multicolumn{2}{c}{ Subset } \\
\cline { 3 - 4 } & & 1 & \\
\hline AUS & 131 & 2.344 & \\
PL & 101 & 3.287 & 5.932 \\
GER & 121 & 3.884 & 1.000 \\
USA & 118 & & \\
Sig. & & 0.087 & \\
\hline
\end{tabular}


Table 9. Oneway ANOVA for the country specific differences between the influential determinants in S1.

\begin{tabular}{|c|c|c|c|c|c|c|}
\hline & & Sum of Squares & df & Mean Square & $\mathrm{F}$ & Sig. \\
\hline \multirow{3}{*}{ Altruism S1 } & Between Groups & 553.914 & 3 & 184.638 & 1.577 & 0.197 \\
\hline & Within Groups & 17558.197 & 150 & 117.055 & & \\
\hline & Total & 18112.110 & 153 & & & \\
\hline \multirow{3}{*}{ Loyalty S1 } & Between Groups & 266.710 & 3 & 88.903 & 2.785 & 0.043 \\
\hline & Within Groups & 4787.687 & 150 & 31.918 & & \\
\hline & Total & 5054.396 & 153 & & & \\
\hline \multirow{3}{*}{ Fairness S1 } & Between Groups & 4015.280 & 3 & 1338.427 & 4.564 & 0.004 \\
\hline & Within Groups & 43992.623 & 150 & 293.284 & & \\
\hline & Total & 48007.903 & 153 & & & \\
\hline \multirow{3}{*}{ Satisfaction S1 } & Between Groups & 1151.850 & 3 & 383.950 & 0.559 & 0.643 \\
\hline & Within Groups & 103074.644 & 150 & 687.164 & & \\
\hline & Total & 104226.494 & 153 & & & \\
\hline \multirow{3}{*}{$\begin{array}{c}\text { Price } \\
\text { Consciousness S1 }\end{array}$} & Between Groups & 5858.031 & 3 & 1952.677 & 3.895 & 0.010 \\
\hline & Within Groups & 75194.878 & 150 & 501.299 & & \\
\hline & Total & 81052.909 & 153 & & & \\
\hline \multirow{3}{*}{ Reference Price S1 } & Between Groups & 207.814 & 3 & 69.271 & 0.524 & 0.666 \\
\hline & Within Groups & 19819.225 & 150 & 132.128 & & \\
\hline & Total & 20027.039 & 153 & & & \\
\hline \multirow{3}{*}{ IncomeS1 } & Between Groups & 2060.094 & 3 & 686.698 & 3.910 & 0.010 \\
\hline & Within Groups & 26342.166 & 150 & 175.614 & & \\
\hline & Total & 28402.260 & 153 & & & \\
\hline \multirow{3}{*}{$\begin{array}{c}\text { Social } \\
\text { Acceptance S1 }\end{array}$} & Between Groups & 57.183 & 3 & 19.061 & 1.057 & 0.369 \\
\hline & Within Groups & 2704.382 & 150 & 18.029 & & \\
\hline & Total & 2761.565 & 153 & & & \\
\hline \multirow{3}{*}{$\begin{array}{c}\text { Social Norm } \\
\text { Compliance S1 }\end{array}$} & Between Groups & 22.030 & 3 & 7.343 & 0.196 & 0.899 \\
\hline & Within Groups & 5615.866 & 150 & 37.439 & & \\
\hline & Total & 5637.896 & 153 & & & \\
\hline \multirow{3}{*}{$\begin{array}{l}\text { High Level of } \\
\text { Reputation S1 }\end{array}$} & Between Groups & 1008.495 & 3 & 336.165 & 3.529 & 0.016 \\
\hline & Within Groups & 14288.544 & 150 & 95.257 & & \\
\hline & Total & 15297.039 & 153 & & & \\
\hline \multirow{3}{*}{ OtherS1 } & Between Groups & 293.321 & 3 & 97.774 & 1.244 & 0.296 \\
\hline & Within Groups & 11787.744 & 150 & 78.585 & & \\
\hline & Total & 12081.065 & 153 & & & \\
\hline
\end{tabular}

Table 10. Kruskal Wallis Test of the country specific between-subjects effects per situation.

\begin{tabular}{ccccccc}
\hline \multicolumn{7}{c}{ Test Statistics $^{\mathrm{a}, \mathrm{b}}$} \\
& Loyalty & Price & Income & Social & High Level of & Loyalty \\
& S1 & Consciousness S1 & S1 & Acceptance S1 & Reputation S1 & S3 \\
\hline Chi-Square & 8.859 & 9.148 & 7.676 & 2.417 & 12.165 & 8.018 \\
df & 3 & 3 & 3 & 3 & 3 & 3 \\
Asymp. Sig. & 0.031 & 0.027 & 0.053 & 0.490 & 0.007 & 0.046 \\
\hline
\end{tabular}

${ }^{\mathrm{a} K r u s k a l}$ Wallis Test; ${ }^{\mathrm{b}}$ Grouping Variable: Country Code Numbers. 
Table 11. Post Hoc Test after Duncan for an assessment of the country specific differences between loyalty in situation 1 .

\begin{tabular}{cccc}
\hline & \multicolumn{2}{c}{ Loyalty S1 } \\
\hline & & \multicolumn{2}{c}{ Duncan } \\
\hline \multirow{2}{*}{ Country Code } & $\mathrm{N}$ & \multicolumn{2}{c}{ Subset for alpha $=0.05$} \\
\cline { 3 - 4 } & & 1 & 2 \\
\hline AUS & 38 & 1.3947 & 2.8571 \\
PL & 28 & 2.8571 & 3.9512 \\
USA & 41 & 3.9512 & 4.8085 \\
GER & 47 & & 0.163 \\
Sig. & & 0.066 & \\
\hline
\end{tabular}

Table 12. Post Hoc Test after Duncan for an assessment of the country specific differences between fairness in situation 1 .

\begin{tabular}{cccc}
\hline & \multicolumn{3}{c}{ FairnessS1 } \\
\hline & \multicolumn{2}{c}{ Duncan } \\
\cline { 3 - 4 } Country Code & $\mathrm{N}$ & \multicolumn{2}{c}{ Subset for alpha $=0.05$} \\
\hline USA & 41 & 8.9756 & \\
AUS & 38 & 10.5263 & 18.6429 \\
PL & 28 & & 20.4894 \\
GER & 47 & & 0.643 \\
Sig. & & 0.697 &
\end{tabular}

Table 13. Post Hoc Test after Duncan for an assessment of the country specific differences between price consciousness in situation 1 .

\begin{tabular}{cccc}
\hline & \multicolumn{3}{c}{ PriceConsciousnessS1 } \\
\hline & \multicolumn{2}{c}{ Duncan } \\
\hline \multirow{2}{*}{ Country Code } & $\mathrm{N}$ & \multicolumn{2}{c}{ Subset for alpha $=0.05$} \\
\hline GER & 47 & 13.7234 & \\
USA & 41 & 19.2683 & 22.2857 \\
PL & 28 & 22.2857 & 30.1842 \\
AUS & 38 & & 0.131 \\
Sig. & & 0.122 & \\
\end{tabular}

Table 14. Post Hoc Test after Duncan for an assessment of the country specific differences between HLoR in situation 1 .

\begin{tabular}{cccc}
\hline & \multicolumn{2}{l}{ High Level of Reputation S1 } \\
\hline & & Duncan & \\
Country Code & $\mathrm{N}$ & \multicolumn{2}{c}{ Subset for alpha $=0.05$} \\
\cline { 3 - 4 } & & 1 & \\
\hline AUS & 38 & 3.1842 \\
GER & 47 & 3.8298 & 9.5366 \\
PL & 28 & 5.0000 & 1.000 \\
USA & 41 & & 0.455 \\
Sig. & & 0.45
\end{tabular}


findings could not be reproduced for S2. None of the 10 influential determinants of intercultural significantly differing influence could be detected in either a one-way ANOVA nor in its nonparametric pendant. Finally, for S3, only one intercultural difference could be found. As illustrated by the Kruskal-Wallis test proofs (because of nonhomogeneous variances, $[\mathrm{F}(3,157)=17.88, p<0.05])$ and the post-hoc test after Duncan, the influential determinant loyalty $[\mathrm{H}(3)=8.02, p$ $<0.05]$ is significantly more important to North Americans than to Germans, Australians or Polish people for S3.

In summary, $\mathrm{H} 3$ could only partially be proven: Regardless of the situational circumstances of the PWYW decision, only loyalty and HLoR significantly differ between cultures in terms of their relative importance to pricing decisions in a PWYW context. When further including the situational factors in the analysis, we found that for S1, loyalty, fairness, PC and HLoR are of differing importance between the tested cultures. However, similar findings could not be reproduced for S2 and S3 and only loyalty proved significantly more important to US Americans for S3 than to participants from Germany, Poland or Australia.

\section{Discussion}

H1 examined the actual influence of situational factors on the customers' WTP. As suggested by the results, the WTP significantly increased when customers did not pay in an anonymous online setting (S1) but actually engaged in face-to-face interaction (S2). However, when the level of social interaction was additionally enhanced by making the pricing decision visible to a third party (friends, S3), the participants' WTP still increased but the effect was not statistically significant. This finding suggests that only the existence of social interaction significantly positively influences a person's WTP in PWYW scenarios, whereas the level of social interaction in the respective situation remains without influence. Gneezy et al. [30] attained comparable findings, which showed that making the amount paid under PWYW circumstances visible to a third party does not significantly increase the WTP. This implies again the self-signalling effects of prices paid under PWYW circumstances. Therefore, the findings suggest that sellers should combine PWYW offers with face-to-face interaction whenever applicable, as the existence of social interaction leads to financially more beneficial outcomes than providing customers with an anonymous online option. Additionally, these findings seem to be country-specific, as a significant increase in the WTP could only be proven for Poland and Australia but not for Germany and the United States. This alteration makes the finding nongeneralizable, as there seem to be interaction effects between the situational circumstances and the cultural backgrounds of the customers in PWYW settings. Thus, when choosing the style of a PWYW price promotion, vendors should consider not only how to offer their products (online vs. face-to-face) but also to whom (i.e., in this case, nationality) they offer PWYW pricing. A possible option would be to offer completely anonymous (online) PWYW options to customers in countries in which the level of social interaction does not significantly influence the WTP, as this type of PWYW promotion will 
lead to an enhanced perception of the vendor in terms of price fairness and at least partially dispenses the customer from cognitive biases.

In addition to dealing with the prices paid under PWYW circumstances, the present study also elaborated on the influential determinants of a PWYW decision. Therefore, $\mathrm{H} 2$ tested whether or not all influential constructs are of identical importance or differ in terms of their influence on a PWYW decision. Overall, we confirmed that the influential determinants not only differ in terms of their relative importance to a PWYW decision but that seven clusters exist, each with significantly different influence on a PWYW decision.

Excluding other potential influences ${ }^{14}$, the determinant loyalty was the least influential construct. As shown by the current research, great uncertainty exists about the influence of loyalty in the PWYW framework, but these results can hardly be interpreted on behalf of current publications: Whereas Marett et al. [7] pointed out that loyal customers are most likely to pay more in participative pricing environments, Kunter [23] referred to loyalty as a situationally restricted construct and Kim et al. [6] did not find significant effects of loyalty on prices paid in PWYW situations. Wieseke, Alavi and Habel [42] noted that loyalty also has an indirect influence on PWYW success, as it can lead to positive word-ofmouth and thus not only influences the PWYW decision itself but also indirectly enhances the business success by motivating others. However, Wieseke et al. [42] also suggested that loyal customers occasionally expect loyalty rewards. From this point of view, the effect of loyalty on a PWYW decision could work counter-intuitively, as loyal customers perceive the PWYW offer as a reward for their loyalty and thus assume they can pay less in such contexts, which is an assumption already made by Schons et al. [24]. However, the minor importance of loyalty in the context of this study can easily be related to the role of the vendor, thus reflecting the view of Kunter [23] and Haumann, Quaiser, Wieseke and Rese [43], who saw loyalty as a multidimensional construct that heavily depends on both situation and vendor characteristics. As the Big Mac represents a product from McDonald's, a globally operating corporation that frequently has image problems and health concerns (According to Azlina, Kamaruzaman, Zetty, Mushaireen, Khazainah, Norzaidah, Norazlina, Noorazlin, Fatimahand Munirah [44] or Marzilli [45]), only few truly loyal McDonald's customers are likely to have participated in this study. Additionally, participants in this context dealt with fictional scenarios, it is most likely that no truly loyal customers actually exist in this case. Accordingly, only 88 out of the 471 participants indicated that they are regular customers of McDonald's, which still does not necessarily make them loyal customers. One can thus expect that the majority of participants were not loyal McDonald's customers, and logically, those participants could not have been influenced by their loyalty to McDonald's, which simply did not exist.

\footnotetext{
${ }^{14}$ The participants infrequently mentioned influential determinants other than the 10 predefined constructs that were presented to them. In addition to this qualitative perspective, from a quantitative absolute point of view, the option other represented the least influential construct, with a mean score of 2.37 ( $\mathrm{SD}=11.05)$ out of 100 using the constant sum approach. Thus, one can assume that the selection of the 10 influential constructs displays all potential influences of a PWYW decision for the time being.
} 
Following this line of thought, also the small influence of HLoR, which belongs to the next influential cluster, can be explained. McDonald's does not represent a company that would typically benefit from its HLoR, as this reputation is rather disadvantageous: Passikoff [46] indicated that Mc Donald's faces image concerns especially within the group of Millenials and Passikoff [47] further outlines Mc Donald's bottom position in brand engagement rankings amongst its fast-food competitors. As noted by Kim et al. [11], page 417, individuals might pay a lower PWYW price if they know that the seller is part of a globally operating chain and can afford losses on PWYW promotions.

In addition to HLoR, the third influential cluster consists of SA, altruism and SNC. With influence comparable to that of HLoR, these determinants are found at the lower end of the influential spectrum of a PWYW decision. With regard to altruism, this finding is in line with the earlier examinations of Kim et al. [6] and Kunter [23], who stated that altruism, does not play a considerable role in PWYW pricing decisions. Likewise, the results of the present study suggest that altruistic considerations are of less importance than other influential determinants to pricing decisions under PWYW circumstances. Jang and Chu [19] discussed the comparably low influence of SNC, stating that injunctive norms (i.e., what ought to be done and thus representing the construct SNC) are not the predominant social norm in a PWYW context. The present study confirmed this finding, as SNC belonged to the influential cluster with a relatively low importance to PWYW decisions. Finally, the affiliation of SA with this influential cluster is more or less in line with earlier PWYW findings: Dorn and Suessmair [22] showed that this construct is influential in PWYW decisions but is located toward the bottom of the ranking of influential determinants. Nonetheless, it remains important to consider the methodological approach when discussing $\mathrm{H} 2$ : As this examination is based on the mean values the participants assigned to the constructs in all three situations, the level of social interaction was not considered in this ranking of influences.

The fourth influential cluster only comprised reference price, thus making it of significantly greater importance to PWYW decisions than loyalty, SA, HLoR, altruism and SNC. This reveals the enhanced importance of reference price on pricing decisions in a PWYW context and is in line with other studies on the influence of reference prices in a PWYW context. Weisstein et al. [37], Armstrong Soule and Madrigal [25] and Chao et al. [20] described the importance of (internal) reference prices for the WTP under PWYW circumstances and showed that reference prices serve as anchors around which most participants indicate their WTP. As no external reference prices were presented or available to the participants of this study, the determinant reference price only comprises a person's internal reference price in this case. However, this only further validates the importance of the overall construct, as even if no external reference prices were available, participants assigned a relatively high importance to their internal reference prices, without external stimuli about the true reference prices. From a managerial perspective, this finding lays a certain importance on the factor price 
knowledge from a customer's point of view: As buyers' range of potential prices in a PWYW context apparently lies around their internal reference price, it is of crucial importance that customers have distinct and true reference prices for the respective products in their mind. As this fact indubitably cannot always be ensured, the presence of external reference prices in the form of suggested prices or regular prices becomes a feasible option for sellers. Studies by Kim et al. [11], Johnson and Cui [17] and Armstrong Soule and Madrigal [25] found the same.

The predominantly greater influence of the two financial influential determinants income and PC is apparent from their affiliation with influential clusters five and six. With a significantly lower influence than PC, income nonetheless belongs to the third most influential cluster for pricing decisions under PWYW circumstances. Unsurprisingly, the WTP under PWYW circumstances is heavily influenced by the participants' individual financial capacities, as noted by Kim et al. [6], Armstrong Soule and Madrigal [25], and Dorn and Suessmair [22]. This implies that individuals base their respective WTP upon their own disposable income when deciding about the amount they are willing to spend in the given context. The age of our participant sample may have enforced the strong position of income in the influential ranking. As noted by León et al. [26], younger customers make smaller payments in PWYW contexts on the one hand because of their lower disposable income and on the other hand because of the fact that younger persons face reduced social expectations to offer high payments. As the underlying sample can be considered relatively young at an average age of 25.6 years, the relative importance of income could be increased because of those two age-related reasons. Younger persons might attribute a higher importance to their relatively low income (in combination with low social expectations) than would older persons, as younger people generally have less financial capacity. This assumption seems to be confirmed when interpreting the relatively high importance participants assigned to the influential determinant income.

Closely connected to the influence of income is the significantly higher position of the determinant PC in the influential ranking. Kunter [23] stated that the influence of PC might be related to individual income, as persons with a low income usually are more price-conscious. Thus, the high relative influence of PC seems a logical consequence that results from the similarly enhanced influence of income. Kim et al. [6] and Marett et al. [7] found this connection and noted that price-conscious customers are mainly driven by the motive of enhanced savings. The results of the present study support the assumption of the high influence of PC by proving that the determinant is associated with the second-most influential cluster for a PWYW pricing decision. The significantly strong influence of the two financial determinants also is informative about the underlying motives to engage in a PWYW pricing process: Consumers who participate in this kind of payment situation are, at least to some extent, interested in making a good bargain. Kunter [23] noted this motive and directly relatedone's obtaining a good bargain to the consumer's characteristic of price-consciousness. This underlying consumer motivation also explains the unsuitability of high priced products for PWYW promo- 
tions [26]. In situations in which a high priced product is offered under PWYW circumstances, the motivation to make a good bargain might outweigh the influence of other determinants, such as the social acceptability of the prices paid or the self-signalling of a PWYW transaction. In the case of low and medium priced products, however, the possibility of making a bargain is fairly limited, which is why other determinants are of greater influence in such situations. Following this train of thought, the results also suggest that financial concerns are not the only driving force in PWYW payment situations, as prices significantly above zero have been paid, which contradicts the sole influence of PC and income.

This assumption is proven, as the influential ranking reveals that fairness is of significantly greater influence than income and of comparable influence to PC. The ranking explains why, despite the large influence of the two financial determinants, in all scenarios, prices significantly above zero were paid. As consumers heavily intended to act fairly toward the company that offered the PWYW pricing, they were highly motivated to pay a fair, adequate and justified price, which puts the cost of producing and selling the product into relation, as the responsibility of setting the price was completely transferred to the buyers. Haws and Bearden [48] referred to this determinant by stating that fairness (from a customer's perspective) disposes of the capacity to moderate and even outweighs the threat of exploitation that sellers often fear when offering PWYW pricing. This assumption has been proven to be correct, as confirmed by the greater relative influence of fairness than then other influential determinants. What additionally is interesting is the fact that even with the tested reference product; fairness still remains one of the most influential motives for a PWYW decision. Participants initially were not expected to act reciprocally and fairly toward McDonald's, as the company itself is often perceived as unethical and unfair [44], [45], [47]. However, internal fairness motives seem to outweigh the negative word-ofmouth with which McDonald's is occasionally associated.

An interesting extension to these findings can be made relative to the research of Schons et al. [24]: The authors not only found that people with high valuation of fairness motives pay higher prices on average but also showed that the preferences for fairness motives in the first (out of multiple) PWYW transactions somewhat constitutes a decision rule upon which subsequent PWYW transactions are based. Thus, the greater the assigned importance of the influential determinant fairness (in the first PWYW transaction), the more likely it is that PWYW pricing will be profitable in the long run, as these fairness concerns form the decision rule upon which future pricing activities for this product in the PWYW context will be made. Therefore, as fairness apparently constitutes one of the most influential determinants in a PWYW pricing context, the long-term profitability of the pricing mechanism can be foreseen by evaluating the price structure and the profitability of short-term PWYW promotions.

Finally, satisfaction was proven to be the most influential determinant, which significantly differs from all other constructs with regard to its relative impor- 
tance to a PWYW pricing decision. This result seems appealing, as in the end, the PWYW pricing mechanism motivates customers to pay what they feel the consumed product is worth. Thus, the greater importance of the determinant can be regarded as a logical consequence that originates from the nature of the pricing mechanism itself and shows PWYW's effectiveness in achieving its original objective: Making people's payments based on their mere satisfaction with a product.

Homburg et al. [39] concluded that satisfied consumers pay more, that satisfaction leads to greater customer loyalty and in the long term, to higher economic returns. In connection with the results of the present study, this implies that satisfaction not only exerts a significant influence on a customer's WTP but that customer satisfaction actually positively influences the buyer's WTP. Thus, the great importance participants assigned to the influential determinant satisfaction also partly explains why prices significantly above zero were paid throughout all scenarios. Furthermore, this finding additionally moderates the expected negative influence on the WTP of the financial constructs income and PC. Therefore, the findings of the present study indicate that consumer satisfaction is the most important criterion to be considered when one offers PWYW promotions and that consumer satisfaction can be a deciding factor in the success of a PWYW promotion. Furthermore, it is important to note that satisfaction in this context not only refers to product characteristics but also to the ambience of the venue and interactions with the staff. Therefore, it is crucial not only to offer great products under PWYW circumstances but also to pay attention to peripheral factors, as in the end, the entire package influences the success of a PWYW offer. Thus, $\mathrm{H} 2$ can holistically be confirmed, as the relative importance of the influential determinants for a PWYW decision significantly differs amongst each other.

$\mathrm{H} 3$ examined the influence of cultural background on the ranking of influential determinants. When neglecting the situational context of a PWYW decision, only loyalty and HLoR have been found to be of differing influence among the tested countries. All other tested constructs were of comparable relevance for the decision process under PWYW circumstances for all participants regardless of their cultural background. The differing importance of the influential determinant HLoR can be related to the culturally differing perceptions customers might have of the seller. In today's globalized world, a globally operating corporation such as McDonald's is perceived differently among various cultures: Whereas for participants from one country, McDonald's might stand for an unethical global firm, individuals from another country might have been totally indifferent about that factor and would not comprehend how this would relate to their pricing decision under PWYW circumstances. Such individuals would thus state that HLoR did not play an influential role in their PWYW pricing decision, whereas it was crucial to participants from another country. Additionally, it is truly interesting that loyalty has a significantly greater influence on the PWYW decisions of North Americans than it has for participants from Australia, Ger- 
many and Poland. Obviously, McDonald's in the United States represents brand customers to which are rather more loyal than are persons in the remaining three tested countries.

Nonetheless, most of the influential determinants did not differ significantly in their relative importance between the tested countries. One possible explanation for this finding can be found in an examination of the respective countries' cultural background according to Hofstede's cultural dimensions: All of the four tested countries belonged to rather Westernized cultures (Figure 2).

All countries have a comparable level of masculinity, whereas in terms of power distance and uncertainty avoidance, only the Polish people differ from the three other countries. Germans tend to be more long-term oriented and with regard to individualism, Australians and US Americans differ from Germans and Poles, but overall, all of the four examined countries are quite comparable with respect to their cultural dimensions, meaning that those countries do not vastly differ in terms of their culture. Thus, it seems logical that the cultural background of the four countries does not significantly influence their respective rankings of the influential determinants.

However, the fact that most of the influential determinants do not differ interculturally in terms of their relative importance to a PWYW decision shows that the influential determinants are mainly robust, regardless of the country in which they are tested and thus show an international applicability of the pricing mechanism for rather Westernized cultures. From a practical point of view, this implies that interculturally comparable settings and designs can be applied when offering PWYW promotions, as the underlying influential motives remain the same and of comparable influence for customers across various Western countries.

\section{Conclusions}

Examination of the data from this study provided several explorative findings for the field of PWYW research. Initially, the research suggested that the existence of social interaction during PWYW transactions had a significantly positive influence on a customer's WTP, whereas the amount of social interaction in nonanonymous PWYW settings remained without a significant influence on the buyer's WTP.

We investigated the influential basis of a PWYW decision and confirmed that

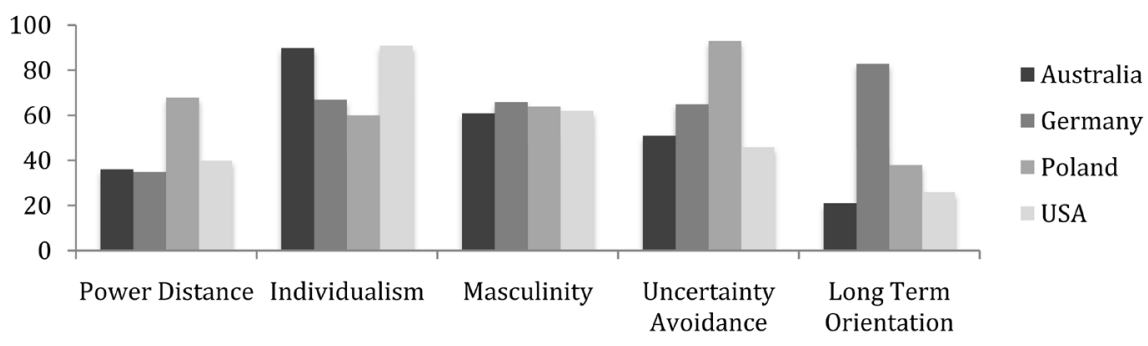

Figure 2. Hofstede's cultural dimensions for the tested countries ${ }^{15}$.

${ }^{15}$ Data retrieved from http://geert-hofstede.com/countries.html, assessed on October $7^{\text {th }}, 2015$. 
the 10 influential determinants, which were deduced from several academic studies that examined the influential basis of PWYW decisions, holistically displayed the entirety of influences upon which PWYW decisions were based. Building upon this, we showed that these influential determinants were of differing relative importance to pricing decisions in the PWYW context and found seven clusters of determinants that varied with regard to their relative influence on PWYW decisions (see Table 15).

Ultimately, we demonstrated that the influential determinants were of relatively robust importance for all participants, regardless of their country of origin, and rejected the assumption of an interculturally differing influential basis of PWYW pricing decisions. This finding highlights the increased important sellers must attribute to the situational circumstances under which they offer PWYW promotions.

\section{Methodological Critique and Outlook for Further Research}

Although most of the hypothetical assumptions employed in this study were confirmed and induced new insights regarding PWYW pricing, the methodo-logical approach of this study had several limitations. The choice of McDonald's Big Mac as a reference product imposed certain drawbacks: As McDonald's represented a globally operating corporation, which was often perceived as unethical or unfair, consumer perceptions regarding the vendor were

Table 15. Overview of the influential clusters and their according contructs, in decending order of importance.

\begin{tabular}{|c|c|c|c|}
\hline \multicolumn{4}{|c|}{ Sub set for alpha $=0.05$} \\
\hline Subset & Construct & Mean Rank Score & Sig. \\
\hline Subset 1 & Satisfaction & 19.86 & 1 \\
\hline \multirow{2}{*}{ Subset 2} & Price Consciousness & 16.63 & \multirow{2}{*}{0.466} \\
\hline & Fairness & 15.96 & \\
\hline Subset 3 & Income & 12.4 & 1 \\
\hline Subset 4 & Reference Price & 8.45 & 1 \\
\hline \multirow{4}{*}{ Subset 5} & Social Norm Compliance & 6.02 & \multirow{4}{*}{0.099} \\
\hline & Altruism & 5.14 & \\
\hline & High Level of Reputation & 5 & \\
\hline & SocialAcceptance & 4.36 & \\
\hline \multirow{4}{*}{ Subset 6} & Altruism & 5.14 & \\
\hline & High Level of Reputation & 5 & \multirow{2}{*}{0.199} \\
\hline & SocialAcceptance & 4.36 & \\
\hline & Loyalty & 3.84 & \\
\hline \multirow{2}{*}{ Subset 7} & Loyalty & 3.84 & 0.107 \\
\hline & Other & 2.37 & \\
\hline
\end{tabular}


somewhat biased and distorted from the outset. Many of the free-text responses confirmed this assumption, as participants frequently indicated that they did not value the Big Mac, that they were concerned about health consequences when purchasing the product or that they did not want to support McDonald's economic claim. These negative perceptions would not only affect the WTP in a PWYW context but also could shape the ranking of the influential determinants, for example, loyalty affiliations were less likely to be associated with McDonald's.

Moreover, the study dealt with hypothetical scenarios and imagined situations. Especially for a product that requires a certain state of mind (e.g., the offer of food or drinks under PWYW conditions requires hunger or at least appetite), the experimental conditions for testing the full potential of the offer are not ideal, as participants do not face the PWYW decision in a suitable moment but at a random point in time. Additionally, the ambience of the venue or the interaction with the staff are assumed to be of crucial influence in PWYW settings but could not realistically be approximated in hypothetical scenarios.

In addition, the measurement of the prices paid in hypothetical PWYW scenarios via the WTP seems critical, as it represents the maximum amount a customer is willing to pay rather than the actual amount paid and can heavily be affected by social approval [7] [39]. Additionally, Schons et al. [24] concluded that prices paid in the first PWYW transaction cannot be considered representative of future transactions, which makes a long-term approach for the assessment of consumer behavior under PWYW indispensable.

Finally, Yu, Yan and Gao [49] concluded that an increase the cognitive load in situations that ask for the WTP significantly reduces the WTP itself. Thus, putting the participants of this study into rather complex scenarios and making them indicate their WTP with a pricing mechanism with which the majority did not have any prior experience may have decreased their WTP.

As this study was of explorative nature and was the first to elaborate holistically on the 10 potential influential determinants and on intercultural differences within the relatively new and innovative field of PWYW pricing, the potential ideas for further research are manifold.

Additional studies that also deal with all 10 influential determinants and confirm that these determinants holistically represent the decisive basis of a PWYW decision is needed and ideally should represent field experiments rather than hypothetical settings. Such studies could then further obtain an influential ranking and confirm the findings of this study that significant differences in the relative importance of the influential determinants prevail. In this context, the connection between the influential determinants and the WTP or the actual prices paid need to be examined. Knowledge of the factors that significantly influence a PWYW decision still does not indicate how they influence the decision. Thus, this aspect needs to be tested to determine whether a direct link between the determinants and WTP exists, how significantly each determinant influences WTP, and what type of changes are elicited by the influential effects of the determinants. 
Moreover, it would be interesting to examine potential interaction effects between the determinants when studying PWYW from a situational perspective. Obviously, the influential determinants are mutually dependent in their influence on a PWYW decision. However, to date nothing has been discovered about these dependencies. Therefore, examination of the connections between the determinants could yield further information about the underlying motives of PWYW decisions.

PWYW research with regard to actual online products is comparably scarce. Examination of the participative pricing mechanism and its effectiveness with actual products (rather than hypothetical settings involving a WTP measurement) in an anonymous online context could deliver further insightful results with regard to the situational circumstances of PWYW offers, consumer behavior in anonymous contexts and the overall applicability of the pricing mechanism.

Finally, little is known about the influence of demographics on consumer behavior in PWYW settings. In addition to an examination of the applicability and effectiveness of the pricing mechanism for various age segments or based upon a buyer's gender, the inclusion of variables such as social class or IQ could yield interesting results. It is highly likely that people with varying (social) backgrounds might respond differently to participative pricing scenarios in which they are given full control about the process of price determination.

\section{References}

[1] Ahmetoglu, G., Furnham, A. and Fagan, P. (2014) Pricing Practices: A Critical Review of Their Effects on Consumer Perceptions and Behaviour. Journal of Retailing and Consumer Services, 21, 696-707.

https://doi.org/10.1016/j.jretconser.2014.04.013

[2] Somervuori, O. (2014) Profiling Behavioral Pricing Research in Marketing. Journal of Product and Brand Management, 23, 462-474.

https://doi.org/10.1108/JPBM-06-2014-0653

[3] Campbell, M.C. (1999) Perceptions of Price Unfairness: Antecedents and Consequences. Journal of Marketing Research, 36, 187-199. https://doi.org/10.2307/3152092

[4] Voelckner, F. and Hofmann, J. (2007) The Price-Perceived Quality Relationship: A Meta-Analytic Review and Assessment of Its Determinants. Marketing Letters, 18, 181-196. https://doi.org/10.1007/s11002-007-9013-2

[5] Xia, L., Monroe, K.B. and Cox, J.L. (2004) The Price Is Unfair! A Conceptual Framework of Price Fairness Perceptions. Journal of Marketing, 68, 1-15. https://doi.org/10.1509/jmkg.68.4.1.42733

[6] Kim, J.Y., Natter, M. and Spann, M. (2009) Pay What You Want: A New Participative Pricing Mechanism. Journal of Marketing, 73, 44-58.

https://doi.org/10.1509/jmkg.73.1.44

[7] Marett, K., Pearson, R. and Moore, R.S. (2012) Pay What You Want: An Exploratory Study of Social Exchange and Buyer Determined Prices of iProducts. Communications of the Association for Information Systems, 30, 1-14.

[8] Spann, M. and Tellis, G.J. (2006) Does the Internet promote Better Consumer Decisions? The Case of Name-Your-Own-Price Auctions. Journal of Marketing, 70, 6578. https://doi.org/10.1509/jmkg.2006.70.1.65 
[9] Kim, J.Y., Natter, M. and Spann, M. (2010) Kish: Where Consumers Pay as They Wish. Review of Marketing Science, 8, 1-12. https://doi.org/10.2202/1546-5616.1118

[10] Kunter, M. (2012) Pay What You Want Pricing: Erfolgsfaktoren, Gestaltungsvariablen, Anwendungsbeispiele [Pay What You Want Pricing: Success Factors, Configuration Elements, Application Fields]. Wirtschaftswissenschaftliches Studium, 41, 302-307. https://doi.org/10.15358/0340-1650-2012-6-302

[11] Kim, J.Y., Natter, M. and Spann, M. (2014) Sampling, Discounts or Pay-WhatYou-Want: Two Field Experiments. International Journal of Research in Marketing, 31, 327-334. https://doi.org/10.1016/j.ijresmar.2014.03.005

[12] Fassnacht, M. and Mahadevan, J. (2010) Grundlagen der Preisfairness: Bestandsaufnahme und AnsätzefürzukünftigeForschung [Basics of Price Fairness: Status Quo and Areas for Future Research]. Journal für Betriebswirtschaft, 60, 295-326. https://doi.org/10.1007/s11301-010-0067-5

[13] Voelckner, F. (2006) Determinanten der Informationsfunktion des Preises: Eine empirische Analyse [Determinats of Theinformationfunction of Prices: An Empiricalanalysis]. Zeitschrift für Betriebswirtschaft, 76, 473-497.

https://doi.org/10.1007/s11573-006-0023-y

[14] Schmidt, K.M., Spann, M. and Zeithammer, R. (2012) Pay What You Want as a Marketing Strategy in Monopolistic and Competitive Markets. Social Science Research Network. http://papers.ssrn.com/sol3/papers.cfm?abstract_id=2191934 https://doi.org/10.2139/ssrn.2191934

[15] Machado, F. and Sinha, R.K. (2015) The Viability of Pay-What-You-Want Pricing. http://ebape.fgv.br/sites/ebape.fgv.br/files/Working-Paper-Fernando-Machado-Via bility-of-Pay-What-you-Want-Pricing.pdf

[16] Gneezy, A., Gneezy, U., Nelson, L.D. and Brown, A. (2010) Shared Social Responsibility: A Field Experiment in Pay-What-You-Want Pricing and Charitable Giving. Science, 329, 325-327. https://doi.org/10.1126/science.1186744

[17] Johnson, J.W. and Cui, A.P. (2013) To Influence or Not to Influence: External Reference Price Strategies in Pay-What-You-Want Pricing. Journal of Business Research, 66, 275-281. https://doi.org/10.1016/j.jbusres.2012.09.015

[18] Greiff, M., Egbert, H. and Xhangolli, K. (2014) Pay What You Want: But Pay Enough! Information Asymmetries and PWYW-Pricing. Management and Marketing. Challenges for the Knowledge Society, 9, 193-204.

[19] Jang, H. and Chu, W. (2012) Are Consumers Acting Fairly toward Companies? An Examination of Pay-What-You-Want Pricing. Journal of Macromarketing, 32, 348 360. https://doi.org/10.1177/0276146712448193

[20] Chao, Y., Fernandez, J. and Nahata, B. (2015) Pay-What-You-Want Pricing: Can It Be Profitable? Journal of Behavioral and Experimental Economics, 57, 176-185. https://doi.org/10.1016/j.socec.2014.09.004

[21] Drevs, F. (2013) The Challenge of the Unknown: The Effect of Pay-What-YouWant on the Market Success of Publicly Subsidized Films. Zeitschrift für Öffentliche und Gemeinwirtschaftliche Unternehmen, 36, 255-270. https://doi.org/10.5771/0344-9777-2013-4-255

[22] Dorn, T. and Suessmair, A. (2016) Is It Really Worth It? A Test of Pay-What-YouWant Pricing Strategies in a German consumer Behaviour Context. Global Business and Economics Review, 18, 82-100. https://doi.org/10.1504/GBER.2016.073321

[23] Kunter, M. (2015) Exploring the Pay-What-You-Want Payment Motivation. Journal of Business Research, 68, 2347-2357.

https://doi.org/10.1016/j.jbusres.2015.03.044 
[24] Schons, L.M., Rese, M., Wieseke, J., Rasmussen, W., Weber, D. and Strotmann, W.C. (2014) There Is Nothing Permanent Except Change: Analyzing Individual Price Dynamics in "Pay-What-You-Want" Situations. Marketing Letters, 25, 25-36. https://doi.org/10.1007/s11002-013-9237-2

[25] Armstrong Soule, C.A. and Madrigal, R. (2015) Anchors and Norms in Anonymous Pay-What-You-Want Pricing Contexts. Journal of Behavioral and Experimental Economics, 57, 167-175. https://doi.org/10.1016/j.socec.2014.10.001

[26] León, F.J., Noguera, J.A. and Sánchez, J.T. (2012) How Much Would You like to Pay? Trust, Reciprocity and Prosocial Motivations in el Trato. Social Science Information, 51, 389-417. https://doi.org/10.1177/0539018412441756

[27] Kim, J.Y., Kaufmann, K. and Stegemann, M. (2014) The Impact of Buyer-Seller Relationships and Reference Prices on the Effectiveness of the Pay What You Want Pricing Mechanism. Marketing Letters, 25, 409-423. https://doi.org/10.1007/s11002-013-9261-2

[28] Mak, V., Zwick, R., Akshay, R.R. and Pattaratanakun, J.A. (2015) "Pay What You Want" as Threshold Public Good Provision. Organizational Behavior and Human Decision Processes, 127, 30-43. https://doi.org/10.1016/j.obhdp.2014.11.004

[29] Jung, M.H., Nelson, L.D., Gneezy, A. and Gneezy, U. (2014) Paying More When Paying for Others. Journal of Personality and Social Psychology, 107, 414-431. https://doi.org/10.1037/a0037345

[30] Gneezy, A., Gneezy, U., Riener, G. and Nelson, L.D. (2012) Pay-What-You-Want, Identity, and Self-Signaling in Markets. Proceedings of the National Academy of Sciences of the United States of America, 109, 7236-7240. https://doi.org/10.1073/pnas.1120893109

[31] Hilbert, L.P. and Suessmair, A. (2015) The Effects of Social Interaction and Social Norm Compliance in Pay-What-You-Want Situations. American Journal of Industrial and Business Management, 5, 548-556. https://doi.org/10.4236/ajibm.2015.58054

[32] Gerpott, T.J. (2017) Pay-What-You-Want Pricing: An Integrative Review of the Empirical Research Literature. Management Science Letters, 7, 35-62.

https://doi.org/10.5267/j.msl.2016.11.004

[33] Cheema, A. and Dholakia, U. (2004) Drivers and Contextual Moderators of Consumer Value Formation in Participative Pricing Mechanisms. Advances in Consumer Research, 31, 46-47.

[34] Voelckner, F. (2006) Methodenzur Messungindividueller Zahlungsbereitschaften: Ein Überblickzum State of the Art [Methods of Measuring the Individual Willingness to Pay: Overview of State of the Art]. Journal für Betriebswirtschaft, 56, 33-60. https://doi.org/10.1007/s11301-006-0002-y

[35] Gautier, P. and van der Klaauw, B. (2012) Selection in a Field Experiment with Voluntary Participation. Journal of Applied Econometrics, 27, 63-84. https://doi.org/10.1002/jae.1184

[36] Mills, P. (2011) Economic and Financial Insights from a Cross-Cultural Pay-WhatYou-Wish Study. Proceedings of the 2011 Annual Meeting of the Academy of Behavioral Finance and Economics, Academy of Behavioral Finance and Economics, Los Angeles, 21-23 September 2011, 107-109.

[37] Weisstein, F.L., Siew, S.W. and Monroe, K.B. (2012) Effects of Product Virtual Experience on Consumers' Willingness to Pay in Participative Pricing Mechanisms. 2012 AMA Winter Educator' Conference: Marketing Theory and Application, American Marketing Association Vol. 23, Chicago, 17-19 August 2012, 266-267.

[38] Miller, K.M., Hofstetter, R., Krohmer, H. and Zhang, Z.J. (2011) How Should Con- 
sumers' Willingness to Pay Be Measured? An Empirical Comparison of State-of-the-Art Approaches. Journal of Marketing Research, 48, 172-184. https://doi.org/10.1509/jmkr.48.1.172

[39] Homburg, C., Koschate, N. and Hoyer, W.D. (2005) Do Satisfied Customers Really Paymore? A Study of the Relationship between Customer Satisfaction and Willingness to Pay. Journal of Marketing, 69, 84-96. https://doi.org/10.1509/jmkg.69.2.84.60760

[40] The Economist (2015) The Big Mac index. http://www.economist.com/content/big-mac-index

[41] Field, A. (2009) Discovering Statistics Using SPSS. 3rd Edition, Sage, London.

[42] Wieseke, J., Alavi, S. and Habel, L. (2014) Willing to Pay More, Eager to Pay less: The Role of Customer Loyalty in Price Negotiations. Journal of Marketing, 78, 1737. https://doi.org/10.1509/jm.13.0104

[43] Haumann, T., Quaiser, B., Wieseke, J. and Rese, M. (2014) Footprints in the Sands of Time: A Comparative Analysis of the Effectiveness of Customer Satisfaction and Customer-Company Identification over Time. Journal of Marketing, 78, 78-102. https://doi.org/10.1509/jm.13.0509

[44] Azlina, S., Kamaruzaman, J., Zetty, M.Z., Mushaireen, M., Khazainah, K., Norzaidah, N., Norazlina, R., Noorazlin, R., Fatimah, A.G. and Munirah, H. (2011) Customer's Perception towards Mcdonald's Icon-Based Nutritional Labels. World Applied Sciences Journal, 12, 1-7.

[45] Marzilli, T. (2015) Mc Donald's Perception Declines but It Remains a Formidable Competitor.

http://www.brandindex.com/article/mcdonalds-perception-declines-it-remains-for $\underline{\text { midable-competitor }}$

[46] Passikoff, R. (2014) Q: What Do You Do When Your Brand Gets Stale? A: Find A Fresh Recipe That Offers More than Just Product.

http://brandkeys.com/q-what-do-you-do-when-your-brand-gets-stale-a-find-a-fres h-recipe-that-offers-more-than-just-product/

[47] Passikoff, R. (2015) Mc Donald's To Eliminate Jobs \& Stores. http://brandkeys.com/mcdonalds-to-eliminate-jobs-stores/

[48] Haws, K.L. and Bearden, W.O. (2006) Dynamic Pricing and consumer Fairness Perceptions. Journal of Consumer Research, 33, 304-311. https://doi.org/10.1086/508435

[49] Yu, X., Yan, B. and Gao, Z. (2014) Can Willingness-to-Pay Values Be Manipulated? Evidence from an Organic Food Experiment in China. Agricultural Economics, 45, 119-127. https://doi.org/10.1111/agec.12134 